\title{
GAUSS-TYPE QUADRATURES FOR WEAKLY SINGULAR INTEGRALS AND THEIR APPLICATION TO FREDHOLM INTEGRAL EQUATIONS OF THE SECOND KIND
}

\author{
HIDEAKI KANEKO AND YUESHENG XU
}

\begin{abstract}
In this paper we establish Gauss-type quadrature formulas for weakly singular integrals. An application of the quadrature scheme is given to obtain numerical solutions of the weakly singular Fredholm integral equation of the second kind. We call this method a discrete product-integration method since the weights involved in the standard product-integration method are computed numerically.
\end{abstract}

\section{INTRODUCTION}

Let $S$ be a subset of $[0,1]$ containing a finite number of points. Define a function associated with $S$ by

$$
\omega_{S}(x)=\inf \{|x-t|: t \in S\} .
$$

For $\alpha>-1$ and a nonnegative integer $k$, a real-valued function $f$ is said to be of $\operatorname{Type}(\alpha, k, S)$ if

$$
\left|f^{(k)}(x)\right| \leq C\left[\omega_{S}(x)\right]^{(\alpha-k)}, \quad x \notin S \text { and } f \in C^{k}([0,1] \backslash S) .
$$

The parameter $\alpha$ is called the index of singularity. For $\alpha>0$, this notation was introduced by Rice in [12]. We remark that if $k=0$ and $-1<\alpha<0$, (1.2) gives

$$
|f(x)| \leq \frac{C}{\left[\omega_{S}(x)\right]^{-\alpha}} \text { for } x \notin S .
$$

In this case, $f$ is not necessarily continuous. In fact, $f$ is allowed to be unbounded at a point in $S$. However, if $\alpha>0$ and $f$ is continuous, then it is easy to verify that $f \in \operatorname{Type}(\alpha, k, S)$ implies that $f$ is an $\alpha$-Hölder continuous function; i.e., $f$ satisfies the following condition [19]:

$$
|f(x)-f(t)| \leq C|x-t|^{\alpha} \text { for } x, t \in[0,1] .
$$

The set of all $\alpha$-Hölder continuous functions is denoted by $\operatorname{Lip}(\alpha)$. For $\alpha>$ -1 , condition (1.2) implies $f \in L_{1}[0,1]$, but $f$ may have weak singularities at some points in $S$. We list some typical functions that belong to $\operatorname{Type}(\alpha, k, S)$ : $f(x)=x^{\alpha}$ and $f(x)=\sin \left(x^{\alpha}\right)$ for $\alpha>-1$ belong to Type $(\alpha, k,\{0\})$ for any

Received by the editor October 31, 1991 and, in revised form, January 25, 1993.

1991 Mathematics Subject Classification. Primary 65D30; Secondary 65D32, 45B05.

Key words and phrases. Gaussian quadrature, singular integrals, weakly singular Fredholm integral equations, product-integration scheme. 
integer $k ; f(x)=\log x$ belongs to Type $(0, k,\{0\})$, whereas $f(x)=x \log x$ belongs to Type $(1, k,\{0\})$ for any integer $k$. One of the goals of this paper is to develop Gauss-type numerical quadratures for the singular integrals

$$
\mathrm{I}(f) \equiv \int_{0}^{1} \rho(x) f(x) d x
$$

where $f \in \operatorname{Type}(\alpha, k, S)$ with $\alpha>-1$ and $\rho$ a weight function that is positive a.e. on $(0,1)$. Throughout this paper, we assume that the integral in (1.4) exists. A classical observation concerning Gaussian quadrature is that the integral (1.4) can be approximated by the sum

$$
\sum_{i=1}^{n} w_{i} f\left(x_{i}\right)
$$

where $\left\{x_{i}\right\}_{i=1}^{n}$ are the zeros of the orthogonal polynomial of degree $n$ with respect to the weight function $\rho$, and that the error of the approximation (1.5) to (1.4) is given by

$$
\frac{\left|f^{(2 n)}(\xi)\right|}{(2 n) !}\left|\int_{0}^{1} \rho(x)\left(x-x_{1}\right)^{2} \cdots\left(x-x_{n}\right)^{2} d x\right| \quad \text { if } f \in C^{(2 n)}[0,1] .
$$

A more detailed discussion of Gaussian quadrature can be found in [2]. For $f \in \operatorname{Type}(\alpha, 2 n, S)$, the error bound given in (1.6) need not hold, since the $(2 n)$ th derivative of $f$ may not be bounded. In $\S 2$, we establish Gauss-type numerical quadrature schemes for integrals (1.4). In $\S 3$, we also derive GaussLegendre-type quadratures for a weakly singular integral, with $\rho \equiv 1$. These results generalize the classical Gaussian quadrature scheme described in (1.5) and (1.6). For numerical quadratures for other types of singular integrals, readers are referred to $[3,9,11,16,17]$, and the references cited within these papers. In $\S 4$, we describe an application of the quadrature scheme obtained in $\S 2$ and $\S 3$ for obtaining numerical solutions of a weakly singular Fredholm integral equations of the second kind. The method described there can be viewed as a discrete product-integration scheme, since the integrals involved in the standard product-integration scheme are computed numerically. Examples are given throughout this paper to illustrate the points being made.

\section{GAUSS-TYPE QUADRATURE}

In this section, we assume $f \in \operatorname{Type}(\alpha, 2 k, S)$ and establish a Gauss-type numerical quadrature for (1.4) by nonlinear spline approximations. First, we consider the case $f \in \operatorname{Type}(\alpha, 2 k,\{0\}) \cap C[0,1]$ with $\alpha>0$ and $\rho \in$ $L_{1}[0,1]$. We define a partition of $[0,1]$, which depends upon the index of singularity $\alpha$, and a piecewise polynomial (or spline) associated with this index. We let $q=\frac{2 k}{\alpha}$ and let $\pi_{\alpha}$ be a partition given by

$$
\begin{aligned}
t_{0} & =0, \\
t_{1} & =n^{-q}, \\
t_{j} & =j^{q} t_{1}, \quad j=2,3, \ldots, n .
\end{aligned}
$$

Clearly, $t_{0}=0, t_{n}=1$, and $t_{0}<t_{1}<\cdots<t_{n}$. On $\left[t_{i}, t_{i+1}\right], 1 \leq i \leq n-1$, let

$$
t_{i} \leq u_{1}^{(i)}<u_{2}^{(i)}<\cdots<u_{k}^{(i)} \leq t_{i+1}
$$


be the $k$ zeros of the orthogonal polynomial $g_{i, k}(x)$ of degree $k$ with respect to the weight function $\rho$ in (1.4); i.e.,

$$
\int_{t_{i}}^{t_{i+1}} \rho(x) g_{i, k}(x) x^{j} d x=0, \quad j=0,1, \ldots, k-1 .
$$

Recall that $\rho$ is positive a.e. on $(0,1)$. Hence, it is positive a.e. on $\left[t_{i}, t_{i+1}\right]$ for each $i=0, \ldots, n-1$. Now, we define a piecewise polynomial $S_{k}$ over $[0,1]$ with knots $\left\{t_{i}\right\}_{i=1}^{n-1}$ by the following rule: $S_{k}(x)$ is the linear interpolant of $f$ at the points $t_{0}$ and $t_{1}$ for $x \in\left[t_{0}, t_{1}\right]$, and $S_{k}(x)$ is the Lagrange interpolant of degree $k-1$ to $f$ at $\left\{u_{j}^{(i)}\right\}_{j=1}^{k}$ for $x \in\left[t_{i}, t_{i+1}\right]$ and for $i=1,2, \ldots, n-1$. Now we use $\mathrm{I}\left(S_{k}\right)$ to approximate $\mathrm{I}(f)$. Let $E_{k}(f) \equiv \mathrm{I}(f)-\mathrm{I}\left(S_{k}\right)$. In order to obtain a bound for the error $E_{k}(f)$, we establish an inequality which is needed later in our investigation.

Lemma 2.1. For $q \geq 1$ and a positive integer $m$, the following inequality holds:

$$
(m+1)^{q}-m^{q} \leq q(m+1)^{q-1} .
$$

Proof. Define the function $\varphi(x)=x^{q}$ on $[m, m+1]$. Then $\varphi$ is continuously differentiable on $[m, m+1]$. Applying the mean value theorem to $\varphi$, we have

$$
(m+1)^{q}-m^{q}=q \xi^{q-1} \quad \text { for some } \xi \in(m, m+1) .
$$

Since the function $x^{q-1}$ is nondecreasing on $(m, m+1)$ for $q \geq 1$, it follows that $\xi^{q-1} \leq(m+1)^{q-1}$. The proof is thus complete.

Theorem 2.2. Let $\rho \in L_{1}[0,1]$ and $\rho>0$ a.e. in $[0,1]$. Let

$$
f \in \operatorname{Type}(\alpha, 2 k,\{0\}) \cap C[0,1]
$$

with $\alpha>0$. Then $\left|E_{k}(f)\right|=\mathscr{O}\left(n^{-2 k+1}\right)$.

Proof. We consider errors contributed from each subinterval. In order to do this, let $E_{k, i}(f) \equiv \int_{t_{i}}^{t_{i+1}} \rho(x) f(x) d x-\int_{t_{i}}^{t_{i+1}} \rho(x) S_{k}(x) d x$, for $i=0,1, \ldots$, $n-1$. First we consider the case $i=0$. Since $S_{k}\left(t_{0}\right)=f\left(t_{0}\right), S_{k}\left(t_{1}\right)=$ $f\left(t_{1}\right), f \in \operatorname{Lip}(\alpha)$, and $S_{k}$ is linear on $\left[t_{0}, t_{1}\right]$, we have

$$
\begin{aligned}
\left|E_{k, 0}(f)\right| & \leq \int_{t_{0}}^{t_{1}} \rho(x)\left|f(x)-S_{k}(x)\right| d x \\
& \leq \int_{t_{0}}^{t_{1}} \rho(x)|f(x)-f(0)| d x+\int_{t_{0}}^{t_{1}} \rho(x)\left|S_{k}(0)-S_{k}(x)\right| d x \\
& \leq C \int_{t_{0}}^{t_{1}} \rho(x) x^{\alpha} d x+\left|S_{k}(0)-S_{k}\left(t_{1}\right)\right| \int_{t_{0}}^{t_{1}} \rho(x) d x \\
& \leq C t_{1}^{\alpha}+\left|f(0)-f\left(t_{1}\right)\right| \int_{t_{0}}^{t_{1}} \rho(x) d x \leq C t_{1}^{\alpha}=C n^{-2 k},
\end{aligned}
$$

where $C$ denotes a generic constant whose value changes from time to time, and which does not depend on $n$.

For $i=1,2, \ldots, n-1$, since $f \in \operatorname{Type}(\alpha, 2 k,\{0\})$, we have $f \in$ $C^{2 k}\left[t_{i}, t_{i+1}\right]$. By (1.6), there exists $\eta_{i} \in\left[t_{i}, t_{i+1}\right]$ such that

$$
\left|E_{k, i}(f)\right|=\frac{\left|f^{(2 k)}\left(\eta_{i}\right)\right|}{(2 k) !}\left|\int_{t_{i}}^{t_{i+1}} \rho(x)\left(x-u_{1}^{(i)}\right)^{2} \cdots\left(x-u_{k}^{(i)}\right)^{2} d x\right| .
$$


Without loss of generality, we may assume $\alpha \leq 2 k$. Thus the function $x^{\alpha-2 k}$ is nonincreasing on $\left[t_{i}, t_{i+1}\right]$. It follows from (1.2) that

$$
\begin{aligned}
\left|E_{k, i}(f)\right| & \leq C\left|\eta_{i}\right|^{\alpha-2 k}\left(t_{i+1}-t_{i}\right)^{2 k} \leq C t_{i}^{\alpha-2 k}\left(t_{i+1}-t_{i}\right)^{2 k} \\
& =C\left(i^{q} t_{1}\right)^{\alpha-2 k}\left[(i+1)^{q}-i^{q}\right]^{2 k} t_{1}^{2 k} .
\end{aligned}
$$

By Lemma 2.1, we have $(i+1)^{q}-i^{q} \leq q(i+1)^{q-1}$. Thus

$$
\left|E_{k, i}(f)\right| \leq C q^{2 k}\left[(i+1)^{2 k(q-1)} i^{q(\alpha-2 k)}\right] t_{1}^{\alpha} .
$$

Note that $q(\alpha-2 k)=\frac{2 k}{\alpha}(\alpha-2 k)=2 k(1-q)$. We then have

$$
(i+1)^{2 k(q-1)} i^{q(\alpha-2 k)}=\left(\frac{i+1}{i}\right)^{2 k(q-1)} \leq 2^{2 k(q-1)} .
$$

Combining (2.3) and (2.4), we obtain

$$
\left|E_{k, i}(f)\right| \leq C q^{2 k} 2^{2 k(q-1)} t_{1}^{\alpha}=C n^{-2 k} .
$$

Finally, it follows that

This completes the proof.

$$
\left|E_{k}(f)\right|=\left|\sum_{i=0}^{n-1} E_{k, i}(f)\right| \leq \sum_{i=0}^{n-1}\left|E_{k, i}(f)\right| \leq C n^{-2 k+1} .
$$

For our second numerical quadrature, we consider the case where $f \in$ Type $(\alpha, 2 k,\{0\})$ with $\alpha>-1$ and $\rho \in L_{\infty}[0,1]$. Let $q=\frac{2 k+1}{\alpha+1}$, and let $\pi_{\alpha}$ be a partition of $[0,1]$ defined by $(2.1)$. Define a piecewise polynomial $S_{k}$ of degree $k-1$ by the rule that $S_{k}(x)=0, x \in\left[t_{0}, t_{1}\right)$, and $S_{k}$ is the Lagrange polynomial of degree $k-1$ to $f$ at $u_{j}^{(i)}$ for $x \in\left[t_{i}, t_{i+1}\right), i=1,2, \ldots, n-2$, and for $x \in\left[t_{n-1}, t_{n}\right], i=n-1$, where $u_{j}^{(i)}$ are the zeros of the orthogonal polynomial of degree $k$ in $\left[t_{i}, t_{i+1}\right]$ with respect to the weight function $\rho$. As in the first case, $\mathrm{I}(f)$ is to be approximated by $\mathrm{I}\left(S_{k}\right)$. The order of convergence of this approximation is provided by the following theorem.

Theorem 2.3. Let $\rho \in L_{\infty}[0,1]$ be positive a.e. in $[0,1]$, and let $\alpha>-1$. Assume that $f \in \operatorname{Type}(\alpha, 2 k,\{0\})$. Then $\left|E_{k}(f)\right|=\mathscr{O}\left(n^{-2 k}\right)$.

Proof. As in the proof of Theorem 2.1, let

$$
E_{k, i}(f) \equiv \int_{t_{i}}^{t_{i+1}} \rho(x) f(x) d x-\int_{t_{i}}^{t_{i+1}} \rho(x) S_{k}(x) d x, \quad i=0,1, \ldots, n-1 .
$$

Then

$$
\begin{aligned}
\left|E_{k, 0}(f)\right| & \leq\|\rho\|_{\infty} \int_{t_{0}}^{t_{1}}\left|f(x)-S_{k}(x)\right| d x \\
& \leq C \int_{t_{0}}^{t_{1}}|f(x)| d x \leq C \int_{t_{0}}^{t_{1}} x^{\alpha} d x \leq C t_{1}^{\alpha+1}=C n^{-2 k-1} .
\end{aligned}
$$

For $i \geq 1$, by using (1.6) and Lemma 2.1, we find

$$
\begin{aligned}
\left|E_{k, i}(f)\right| & \leq \frac{\left|f^{(2 k)}\left(\eta_{i}\right)\right|}{(2 k) !}\left|\int_{t_{i}}^{t_{i+1}} \rho(x)\left(x-u_{1}^{(i)}\right)^{2} \cdots\left(x-u_{k}^{(i)}\right)^{2} d x\right| \\
& \leq\left|\eta_{i}\right|^{\alpha-2 k}\left(t_{i+1}-t_{i}\right)^{2 k+1} \leq C t_{i}^{\alpha-2 k}\left(t_{i+1}-t_{i}\right)^{2 k+1} \\
& =C\left(i^{q} t_{1}\right)^{\alpha-2 k}\left[(i+1)^{q}-i^{q}\right]^{2 k+1} t_{1}^{2 k+1} \\
& \leq C q^{2 k+1}(i+1)^{(q-1)(2 k+1)} i^{q(\alpha-2 k)} t_{1}^{\alpha+1} .
\end{aligned}
$$


Since $q(\alpha-2 k)=q(\alpha+1)-q(2 k+1)=(2 k+1)-q(2 k+1)=(1-q)(2 k+1)$, as proved in (2.4), we have the following estimate:

$$
\left|E_{k, i}(f)\right| \leq C q^{2 k+1} 2^{(q-1)(2 k+1)} t_{1}^{\alpha+1} \leq C n^{-2 k-1} .
$$

Therefore,

$$
\left|E_{k}(f)\right|=\left|\sum_{i=0}^{n-1} E_{k, i}(f)\right| \leq C n^{-2 k} .
$$

This completes the proof.

The degree of approximation of the second of the above methods is of order $\frac{1}{n}$ smaller than that of the first method, owing to more smoothness of the weight function $\rho(x)$. Before we describe generalizations of Theorems 2.2 and 2.3 , it is useful to recall the product-integration scheme of [10] and [16], which can be used to approximate integrals of the form

$$
\mathrm{I}(f)=\int_{-1}^{1} \rho(x) f(x) d x
$$

for certain classes of weight functions $\rho$. Here we take $x_{n i} \quad(1 \leq i \leq n, n \geq 1)$ to be the set of Chebyshev points, i.e., the zeros of the $n$ th-degree Chebyshev polynomial of the first kind. By $L_{n}^{f}(x)$ we denote the Lagrange polynomial of degree less than $n$ that interpolates $f$ at $x_{n i}$. The integral in (2.5) is now approximated by

$$
\mathrm{I}_{n}(f)=\int_{-1}^{1} \rho(x) L_{n}^{f}(x) d x=\sum_{i=1}^{n} w_{n i} f\left(x_{n i}\right),
$$

where $w_{n i}=\int_{-1}^{1} \rho(x) l_{n i}(x) d x$ and $l_{n i}$ is the fundamental Lagrange polynomial of degree $n-1$ such that $l_{n i}\left(x_{n j}\right)=\delta_{i j}$. With the aid of the Christoffel-Darboux identity, we obtain

$$
w_{n i}=\left(\frac{2}{n}\right)\left[\frac{1}{2} a_{0}+\sum_{k=1}^{n-1} a_{k} \cos \left(k \cos ^{-1} x_{n i}\right)\right],
$$

where

$$
a_{k}=\int_{-1}^{1} \rho(x) T_{k}(x) d x,
$$

and $T_{k}$ is the $k$ th-degree Chebyshev polynomial of the first kind. Piessens and Branders [10] developed recursion formulas for a few choices of $\rho(x)$ to evaluate the number $a_{k}$ in (2.8). These values are subsequently used to compute $w_{n i}$. The order of approximation of the product-integration scheme is given by

$$
\left|\mathrm{I}(f)-\mathrm{I}_{n}(f)\right| \leq\left(\frac{\pi}{2}\right)^{k} \frac{\left\|f^{(k)}\right\|_{\infty}}{n(n-1) \cdots(n-k+1)},
$$

provided that $f \in C^{(k)}[-1,1]$ and $n \geq k+1$.

Theorems 2.2 and 2.3 generalize the method of [10] and [16] in the following sense. First, in order to obtain the order of the approximation given by (2.9), it is necessary that $f$ be sufficiently smooth as indicated in (2.9). Second, in the product-integration method, the weights in (2.7) depend upon the function $\rho$. 
Also, in many examples which are suited for the product-integration method of (2.5), the weight functions can be included as a part of the function $f$ in (1.4). More specifically, in Example 3.2 below, we consider the integral

$$
\int_{0}^{1} \frac{\sqrt{\left|x-\frac{1}{2}\right|}}{\sqrt{|\lambda-x|}} d x, \quad \text { with } \lambda=1 .
$$

To apply the current method, we let $\rho \equiv 1$ and $f(x)=\sqrt{\left|x-\frac{1}{2}\right|} / \sqrt{|\lambda-x|}$ in (1.4). Subsequently, we can use Gaussian quadrature, for which the weights are already tabulated. Examples supporting these assertions will be given in the next section.

Next, we extend the results obtained in the first part of this section to more general cases, where $S$ contains more than one point. Assume $S=\left\{0=s_{1}<\right.$ $\left.s_{2}<\cdots<s_{m}<s_{m+1}=1\right\}$. Let $f \in \operatorname{Type}(\alpha, 2 k, S)$. Set

$$
\begin{aligned}
\tau_{2 i-1} & =s_{i}, \quad i=1,2, \ldots, m+1, \\
\tau_{2 i} & =\frac{1}{2}\left(s_{i}+s_{i+1}\right), \quad i=1,2, \ldots, m .
\end{aligned}
$$

In each interval $\left[\tau_{i}, \tau_{i+1}\right]$, the function $f^{(k)}(x)$ has only one singularity. Namely, a singularity is located at $\tau_{i}$ if $i$ is odd and at $\tau_{i+1}$ if $i$ is even. In the ensuing discussion, we assume that $f^{(k)}$ has a singularity at the left endpoint $\tau_{i}$. The case for which $f^{(k)}$ has a singularity at the right endpoint $\tau_{i+1}$ can be handled similarly. Let

$$
t \equiv \frac{x-\tau_{i}}{\tau_{i+1}-\tau_{i}}
$$

Then by change of variables, we obtain

$$
\int_{\tau_{i}}^{\tau_{i+1}} \rho(x) f(x) d x=\left(\tau_{i+1}-\tau_{i}\right) \int_{0}^{1} \rho\left(\tau_{i}+\left(\tau_{i+1}-\tau_{i}\right) t\right) f\left(\tau_{i}+\left(\tau_{i+1}-\tau_{i}\right) t\right) d t .
$$

Denote

$$
\hat{\rho}(t)=\rho\left(\tau_{i}+\left(\tau_{i+1}-\tau_{i}\right) t\right) \text { and } \hat{f}(t)=f\left(\tau_{i}+\left(\tau_{i+1}-\tau_{i}\right) t\right) .
$$

If $\alpha>0, \rho \in L_{1}[0,1]$ and $f \in \operatorname{Type}(\alpha, 2 k, S) \cap C[0,1]$, then $\hat{\rho} \in L_{1}[0,1]$ and $\hat{f} \in \operatorname{Type}(\alpha, 2 k,\{0\}) \cap C[0,1]$. Applying the first quadrature scheme of Theorem 2.2 to

$$
\hat{\mathbf{I}}(\hat{f})=\int_{0}^{1} \hat{\rho}(x) \hat{f}(x) d x,
$$

we obtain an order of convergence $\mathscr{O}\left(n^{-2 k+1}\right)$. If $\alpha>-1, \rho \in L_{\infty}[0,1]$, and $f \in \operatorname{Type}(\alpha, 2 k, S)$, then $\hat{\rho} \in L_{\infty}[0,1]$ and $\hat{f} \in \operatorname{Type}(\alpha, 2 k,\{0\})$. Now applying the second quadrature scheme of Theorem 2.3 to (2.10), we obtain an order of convergence $\mathscr{O}\left(n^{-2 k}\right)$. We summarize the above discussion in the next theorem.

Theorem 2.4. (i) Let $\rho \in L_{1}[0,1], \rho>0$ a.e. in $[0,1]$, and $f \in \operatorname{Type}(\alpha, 2 k, S)$ $\cap C[0,1]$ with $\alpha>0$. Then the error of the first quadrature scheme described above is given by $\left|E_{k}(f)\right|=\mathscr{O}\left(n^{-2 k+1}\right)$. 
(ii) Let $\rho \in L_{\infty}[0,1], \rho>0$ a.e. in $[0,1]$, and $f \in \operatorname{Type}(\alpha, 2 k, S)$ with $\alpha>-1$. Then the error of the second scheme is given by $\left|E_{k}(f)\right|=\mathscr{O}\left(n^{-2 k}\right)$.

Remark. As indicated in Theorem 2.4, the orders of accuracy of the quadrature schemes are independent of $\alpha$. Thus, it is not necessary for $f$ to have the same degree of singularity at all the points in $S$. This is demonstrated in an example given at the end of the next section.

\section{GAUSS-LEGENDRE-TYPE QUADRATURES}

In this section we shall develop a scheme which will be called Gauss-Legendretype quadrature. In practice, one of the most interesting cases of Theorem 2.3 is when the weight function $\rho(x) \equiv 1$ on $[0,1]$. In this case, our Gauss-type quadrature scheme of Theorem 2.3 can be simplified. We consider the following integral

$$
\mathrm{I}(f)=\int_{0}^{1} f(x) d x
$$

where $f \in \operatorname{Type}(\alpha, 2 k, S)$ with $\alpha>-1$.

Since $\rho \equiv 1$, the zeros of the Legendre polynomial

$$
g_{k}(x)=\frac{1}{2^{k} k !} \frac{d^{k}}{d x^{k}}\left(x^{2}-1\right)^{k}, \quad x \in[-1,1],
$$

of degree $k$ will be used as the interpolation points. As before, we assume $S=\{0\}$ without loss of generality. Let $q=\frac{2 k+1}{\alpha+1}$. Define a partition $\pi_{\alpha}$ of $[0,1]$ as in $\S 2$, i.e.,

$$
\pi_{\alpha}: t_{0}=0, t_{1}=n^{-q}, t_{j}=j^{q} t_{1}, \quad j=2,3, \ldots, n .
$$

Now we use

$$
\hat{\mathrm{I}}(f)=\int_{t_{1}}^{1} f(x) d x=\sum_{i=1}^{n-1} \int_{t_{i}}^{t_{i+1}} f(x) d x
$$

to approximate $\mathbf{I}(f)$. In order to evaluate $\hat{\mathrm{I}}(f)$, we introduce a mapping

$$
s=\frac{2 x-\left(t_{i+1}+t_{i}\right)}{t_{i+1}-t_{i}},
$$

which maps $\left[t_{i}, t_{i+1}\right]$ to $[-1,1]$. Hence

$$
\hat{\mathrm{I}}(f)=\int_{-1}^{1} F(s) d s
$$

where

$$
F(s)=\sum_{i=1}^{n-1} \frac{1}{2}\left(t_{i+1}-t_{i}\right) f\left(\frac{1}{2}\left(t_{i+1}-t_{i}\right) s+\frac{1}{2}\left(t_{i+1}+t_{i}\right)\right) .
$$

Assume that $u_{i}(i=1,2, \ldots, k)$ are the zeros of Legendre polynomial of degree $k$. Let $S_{k}$ be the Lagrange interpolation polynomial of degree $k-1$ which interpolates $F$ at $u_{i}$. Then

$$
S_{k}(x)=\sum_{i=1}^{k} F\left(u_{i}\right) l_{i}(x),
$$


where $l_{i}$ are the Lagrange fundamental polynomials of degree $k-1$ defined by

$$
l_{i}(x)=\prod_{\substack{j=1 \\ j \neq i}}^{k} \frac{x-u_{j}}{u_{i}-u_{j}}
$$

In (3.6), we replace $F(s)$ by $S_{k}(s)$ to obtain

$$
\hat{\mathrm{I}}\left(S_{k}\right)=\sum_{i=1}^{k} w_{i} F\left(u_{i}\right), \quad \text { where } w_{i}=\int_{-1}^{1} l_{i}(x) d s .
$$

By applying Theorem 2.3, we have the following theorem concerning the accuracy of our Gauss-Legendre-type quadrature scheme.

Theorem 3.1. Let $f \in \operatorname{Type}(\alpha, 2 k,\{0\})$ with $\alpha>-1$. Let $E_{k}(f)=\mathrm{I}(f)-$ $\hat{\mathrm{I}}\left(S_{k}\right)$, where $\mathrm{I}(f)$ and $\hat{\mathrm{I}}\left(S_{k}\right)$ are defined by (3.1) and (3.4), respectively. Then $\left|E_{k}(f)\right|=\mathscr{O}\left(n^{-2 k}\right)$.

Proof. Note that

$$
\begin{aligned}
\hat{\mathrm{I}}\left(S_{k}\right) & =\int_{-1}^{1} S_{k}(x) d x=\int_{-1}^{1} \sum_{i=1}^{k} F\left(u_{i}\right) l_{i}(x) d x \\
& =\int_{-1}^{1} \sum_{i=1}^{k} \sum_{j=1}^{n-1} \frac{1}{2}\left(t_{j+1}-t_{j}\right) f\left(\frac{1}{2}\left(t_{j+1}-t_{j}\right) u_{i}+\frac{1}{2}\left(t_{j+1}+t_{j}\right)\right) l_{i}(x) d x \\
& =\sum_{j=1}^{n-1} \frac{1}{2}\left(t_{j+1}-t_{j}\right) \sum_{i=1}^{k} \int_{-1}^{1} f\left(\frac{1}{2}\left(t_{j+1}-t_{j}\right) u_{i}+\frac{1}{2}\left(t_{j+1}+t_{j}\right)\right) l_{i}(x) d x
\end{aligned}
$$

Let

$u_{i}^{(j)}=\frac{1}{2}\left(t_{j+1}-t_{j}\right) u_{i}+\frac{1}{2}\left(t_{j+1}+t_{j}\right), \quad i=1,2, \ldots, k, j=1,2, \ldots, n-1$.

Then

$$
\begin{aligned}
\hat{\mathrm{I}}\left(S_{k}\right) & =\sum_{j=1}^{n-1} \sum_{i=1}^{k} \int_{t_{j}}^{t_{j+1}} f\left(u_{i}^{(j)}\right) l_{i}\left(\frac{2 x-\left(t_{j+1}+t_{j}\right)}{t_{j+1}-t_{j}}\right) d x \\
& =\sum_{j=1}^{n-1} \int_{t_{j}}^{t_{j+1}} \sum_{i=1}^{k} f\left(u_{i}^{(j)}\right) \prod_{p=1, p \neq i}^{k} \frac{x-u_{p}^{(j)}}{u_{i}^{(j)}-u_{p}^{(j)}} d x .
\end{aligned}
$$

Define a piecewise polynomial of degree $k-1$ by $\widehat{S}_{k}(x)=0$, for $x \in\left[t_{0}, t_{1}\right]$ and

$$
\widehat{S}_{k}(x)=\sum_{i=1}^{k} f\left(u_{i}^{(j)}\right) \prod_{\substack{p=1 \\ p \neq i}}^{k} \frac{x-u_{p}^{(j)}}{u_{i}^{(j)}-u_{p}^{(j)}} \text { for } x \in\left[t_{i}, t_{i+1}\right),
$$

where $i=1,2, \ldots, n-2$, and for $x \in\left[t_{n-1}, t_{n}\right]$ if $i=n-1$. Thus, we can rewrite $\hat{\mathrm{I}}\left(S_{k}\right)$ as follows,

$$
\hat{\mathrm{I}}\left(S_{k}\right)=\int_{t_{1}}^{t_{n}} \widehat{S}_{k}(x) d x=\mathrm{I}\left(\widehat{S}_{k}\right) .
$$

Applying Theorem 2.3, we obtain $\left|E_{k}(f)\right|=\left|\mathrm{I}(f)-\mathrm{I}\left(\widehat{S}_{k}\right)\right|=\mathscr{O}\left(n^{-2 k}\right)$.

The above quadrature formula and Theorem 3.1 can be extended to the general case where $S$ contains more than one point, as we have done in $\S 2$. 


\section{Numerical examples.}

Example 3.1. In this example, we compare our method of Theorem 3.1 with the Gauss-Chebyshev integration rule of the first kind ([3, p. 98]),

$$
\begin{aligned}
& \int_{-1}^{1}\left(1-x^{2}\right)^{-1 / 2} f(x) d x \\
& \quad=\frac{\pi}{n} \sum_{i=1}^{n} f\left(x_{i}\right)+\frac{\pi}{2^{2 n-1}(2 n) !} f^{(2 n)}(\xi), \quad-1<\xi<1,
\end{aligned}
$$

where $x_{i}=\cos \left(\frac{(2 i-1) \pi}{2 n}\right)$ for $i=1, \ldots, n$. Consider

$$
\int_{-1}^{1} \frac{1}{\sqrt{|x|}} d x \text {. }
$$

Of course, we use $f(x)=\sqrt{1-x^{2}} / \sqrt{|x|}$ in (3.8). For our present method, the linear $(k=2, q=10)$ and the quadratic $(k=3, q=14)$ polynomials are tested. The following data list the respective quadrature errors and their decay exponents (briefly, decay exp.). Each decay exponent is computed by $\frac{\ln \left(e_{n} / e_{m}\right)}{\ln (m / n)}$, where $e_{n}$ denotes the error of the approximation for the value $n$. Notice the inadequate performance of the Gauss-Chebyshev method in the case of nonsmooth $f$ in (3.8).

\begin{tabular}{ccccc}
\multicolumn{5}{c}{ Data 1: present method } \\
$n$ & Errors $(k=2)$ & decay exp. & Errors $(k=3)$ & decay exp. \\
8 & $2.23 E-1$ & & $9.22 E-2$ & \\
16 & $3.38 E-2$ & 2.72 & $6.64 E-3$ & 3.80 \\
32 & $3.21 E-3$ & 3.40 & $2.87 E-4$ & 4.53 \\
64 & $2.40 E-4$ & 3.74 & $6.11 E-6$ & 5.55
\end{tabular}

DAtA 2: Gauss-Chebyshev method

$$
\begin{array}{cc}
n & \text { Errors } \\
8 & 7.47 E-1 \\
16 & 5.33 E-1 \\
32 & 3.78 E-1 \\
64 & 2.34 E-1
\end{array}
$$

There are numerous other quadratures of Gauss type in which an integral of the form $\int_{a}^{b} w(x) f(x) d x$ is approximated by a sum of the form $\sum_{k=1}^{n} w_{k} f\left(x_{k}\right)$, e.g., the Gauss-Chebyshev rule of the second kind $([3$, p. 98]), the method of 
A. Young ([3, p. 176]), etc. Additional numerical experiments indicate that none of these methods performs adequately in the case of nonsmooth function $f$. The integral in the above example can also be dealt with by the method of Stenger [18].

Example 3.2. In this example we compare the present method with the productintegration method of [10] and [16]. We consider

$$
\int_{0}^{1} \frac{\sqrt{\left|x-\frac{1}{2}\right|}}{\sqrt{1-x}} d x
$$

We evaluate this integral by computing

$$
\int_{0}^{1 / 2} \frac{\sqrt{\frac{1}{2}-x}}{\sqrt{1-x}} d x+\int_{1 / 2}^{3 / 4} \frac{\sqrt{x-\frac{1}{2}}}{\sqrt{1-x}} d x+\int_{3 / 4}^{1} \frac{\sqrt{x-\frac{1}{2}}}{\sqrt{1-x}} d x,
$$

where the integrands in the first two integrals belong to Type $\left(\frac{1}{2}, 2 k,\left\{\frac{1}{2}\right\}\right)$ and the one in the third belongs to Type $\left(-\frac{1}{2}, 2 k,\{1\}\right)$ for each $k$. The integer $n$ represents the total number of knots taken over $[0,1]$. The number of knots are distributed proportionately to the lengths of intervals so that $\frac{n}{2}$ were used for the first integral and $\frac{n}{4}$ for the second and the third integrals. Piecewise linear polynomials were used. The exact value is 1.051818151074224 .

\begin{tabular}{ccc}
\multicolumn{3}{c}{ Data 1: present method } \\
$n$ & Errors & Decay exp. \\
16 & $4.68 E-3$ & \\
32 & $4.45 E-4$ & 3.39 \\
64 & $3.58 E-5$ & 3.63 \\
128 & $2.54 E-6$ & 3.81
\end{tabular}

DATA 2: product-integration method

$\begin{array}{ccc}n & \text { Errors } & \text { Decay ex } \\ 16 & 5.30 E-3 & \\ 32 & 1.87 E-3 & 1.50 \\ 64 & 6.64 E-4 & 1.50 \\ 128 & 2.35 E-4 & 1.50\end{array}$

Because of the recursion formula used to compute the weights, the productintegration method required more CPU time to execute than the current method. As with the Gauss-Chebyshev method of Example 1, the product-integration method of (2.6) does not perform adequately in the case of nonsmooth $f$. 


\section{DisCRETE PRODUCT-INTEGRATION SCHEME}

In this section, we describe an application of the quadrature methods in $\S 2$ and $\S 3$ to obtaining numerical solutions of weakly singular Fredholm integral equations of the second kind. The particular numerical method which we use is product-integration. To familiarize the reader with the problem, we give a brief introduction to the product-integration method. The equation in which we are interested is given by

$$
\varphi(x)-\int_{0}^{1} g_{\alpha}(|x-y|) m(x, y) \varphi(y) d y=f(x), \quad 0 \leq x \leq 1,
$$

where $g_{\alpha}, m$, and $f$ are known functions and $\varphi$ is the function to be determined. Moreover, $m$ represents the smooth part of the kernel, and we assume that $g_{\alpha}$ is of the following form:

$$
g_{\alpha}(|x-y|)= \begin{cases}|x-y|^{\alpha}, & -1<\alpha<0 \\ \log |x-y|, & \alpha=0\end{cases}
$$

The equations of the form (4.1) with kernel (4.2) arise in many interesting applications, e.g., [5] and [7]. Regularity properties of the solutions of equation (4.1) were studied by Graham [4], Richter [13], and Schneider [14]. That the result of Schneider can be extended to hold for weakly singular Hammerstein equations was shown in [6]. Writing $K \varphi(x) \equiv \int_{0}^{1} g_{\alpha}(|x-y|) m(x, y) \varphi(y) d y$, equation (4.1) becomes

$$
\varphi-K \varphi=f .
$$

Denote $q=\frac{k}{\alpha+1}$, where $k$ is a positive integer. We let

$$
\begin{array}{llrl}
x_{j, n} & =\frac{1}{2}\left(\frac{2 j}{n}\right)^{q}, & & 0 \leq j \leq \frac{n}{2}, \\
x_{j, n} & =1-x_{n-j, n}, & & \frac{n}{2} \leq j \leq n,
\end{array}
$$

and let $x_{j, n}<s_{1, j}<s_{2, j}<\cdots<s_{k, j}<x_{j+1, n}$ be any $k$ distinct points in $\left[x_{j, n}, x_{j+1, n}\right]$ for each $j=0,1, \ldots, n-1$. Approximate $\varphi$ by $\varphi_{n}$ whose restriction to $\left[x_{j, n}, x_{j+1, n}\right]$ is the polynomial of degree $k-1$ interpolating $\varphi$ at $\left\{s_{i, j}\right\}_{i=1}^{k}$. Upon replacing $\varphi$ in (4.3) by $\varphi_{n}$, we obtain an approximate equation

$$
\varphi_{n}(x)-\sum_{j=0}^{n-1} \sum_{i=1}^{k} \varphi_{n}\left(s_{i, j}\right) \int_{x_{j, n}}^{x_{j+1, n}} g_{\alpha}(|x-y|) m(x, y) l_{i j}(y) d y=f(x),
$$

where

$$
l_{i j}(y)=\prod_{\substack{p=1 \\ p \neq i}}^{k} \frac{y-s_{p, j}}{s_{i, j}-s_{p, j}} \text { for } y \in\left[x_{j, n}, x_{j+1, n}\right] .
$$

Equation (4.5) can be solved by letting $x=s_{p, r}$ for $0 \leq r \leq n-1$ and $1 \leq p \leq k$. Denote

$$
w_{i j n}(x) \equiv \int_{x_{j, n}}^{x_{j+1, n}} g_{\alpha}(|x-y|) m(x, y) l_{i j}(y) d y,
$$


and

$$
K_{n} \varphi(x) \equiv \sum_{j=0}^{n-1} \sum_{i=1}^{k} w_{i j n}(x) \varphi\left(s_{i, j}\right) .
$$

Equation (4.5) now becomes

$$
\varphi_{n}-K_{n} \varphi_{n}=f \text {. }
$$

A main characteristic of the product-integration scheme is that the weights (4.6) are computed exactly. However, in practice, this may not be an easy task to carry out, because of the complexity involved in the form of $m$. The discrete product-integration scheme is the product-integration scheme in which the weights (4.6) are computed numerically. The product-integration scheme of Piessens and Branders, and that of Sloan, can be used to accomplish this. But, a slight modification in the form of the kernel makes the recursion formulas developed in [10] inapplicable. Also, an application of a more direct and efficient numerical quadrature scheme to evaluate (4.6) is less expensive computationally than applying the product-integration scheme as pointed out earlier. In this paper, we apply the Gauss-Legendre-type quadrature of Theorem 3.1 to compute (4.6).

A standard argument $\left[8\right.$, p. 176] shows that $\left\{K_{n}\right\}_{n=1}^{\infty}$, with each $K_{n}$ viewed as a linear operator of $C[0,1]$ into itself, is a sequence of collectively compact operators which converges pointwise to $K$ in $C[0,1]$. For the theory of collectively compact operators, readers are referred to [1]. Moreover, Schneider [15] showed that

$$
\left\|\varphi-\varphi_{n}\right\|_{\infty}=\mathscr{O}\left(n^{-k}\right)
$$

We denote by $w_{i j n}^{*}(x)$ the numerically computed value of (4.6) using the GaussLegendre-type quadrature. By virtue of Theorem 3.1, we find

$$
\left|w_{i j n}(x)-w_{i j n}^{*}(x)\right|=\mathscr{O}\left(n^{-2 k}\right)
$$

for each $x \in[0,1], 1 \leq i \leq k$, and $0 \leq j \leq n-1$. Let

$$
K_{n}^{*} \varphi(x) \equiv \sum_{j=0}^{n-1} \sum_{i=1}^{k} w_{i j n}^{*}(x) \varphi\left(s_{i, j}\right),
$$

so that the discrete product-integration method can be written as

$$
\varphi_{n}^{*}-K_{n}^{*} \varphi_{n}^{*}=f .
$$

By evaluating both sides of equations (4.8) and (4.12) at $\left\{s_{i, j}\right\}$, we obtain respectively the corresponding matrix equations,

$$
\bar{\varphi}_{n}-\bar{K}_{n} \bar{\varphi}_{n}=\bar{f}
$$

and

$$
\bar{\varphi}_{n}^{*}-\bar{K}_{n}^{*} \bar{\varphi}_{n}^{*}=\bar{f}
$$

where

$$
\begin{aligned}
\bar{f} & =\left(f_{i, j}\right) \\
& =\left(f\left(s_{1,0}\right), \ldots, f\left(s_{k, 0}\right), f\left(s_{1,1}\right), \ldots, f\left(s_{k, 1}\right), \ldots, f\left(s_{k, n-1}\right)\right)^{T},
\end{aligned}
$$




$$
\bar{\varphi}_{n}=\left(\varphi_{n}\left(s_{1,0}\right), \ldots, \varphi_{n}\left(s_{k, 0}\right), \varphi_{n}\left(s_{1,1}\right), \ldots, \varphi_{n}\left(s_{k, 1}\right), \ldots, \varphi\left(s_{k, n-1}\right)\right)^{T},
$$

and

$$
\bar{K}_{n} \equiv\left(w_{i j n}\left(s_{p, q}\right)\right) \quad \text { for } 1 \leq i, p \leq k \text { and } 0 \leq j, q \leq n-1,
$$

is a $(k n) \times(k n)$ matrix. Also, $\bar{\varphi}_{n}^{*}$ and $\bar{K}_{n}^{*}$ are obtained similarly by replacing $\varphi_{n}$ in (4.16) and $w_{i j n}$ in (4.17) by $\varphi_{n}^{*}$ and $w_{i j n}^{*}$, respectively. We are now in a position to prove the main result in this section.

Theorem 4.1. Let $\varphi_{n}$ and $\varphi_{n}^{*}$ be the solutions of (4.8) and (4.12), respectively. Then $\left\|\varphi_{n}-\varphi_{n}^{*}\right\|_{\infty}=\mathscr{O}\left(n^{-2 k+1}\right)$.

Proof. Since $\left\{K_{n}\right\}_{n=1}^{\infty}$ is a sequence of collectively compact operators converging pointwise to $K$ in $C[0,1]$, an application of the Banach-Steinhaus theorem [1] shows that

$$
\sup _{n} \sum_{i=1}^{k} \sum_{j=0}^{n-1}\left|w_{i j n}(s)\right|<\infty \quad \text { for each } s \in[0,1] .
$$

Let $\|\cdot\|_{\infty}$ denote the matrix norm associated with the supremum norm on $R^{k n}$. Then by the definitions of the matrices $\bar{K}_{n}$ and $\bar{K}_{n}^{*}$, we have

$$
\left\|\bar{K}_{n}-\bar{K}_{n}^{*}\right\|_{\infty}=\max _{\substack{1 \leq p \leq k \\ 0 \leq q \leq n-1}} \sum_{i=1}^{k} \sum_{j=0}^{n-1}\left|w_{i j n}\left(s_{p, q}\right)-w_{i j n}^{*}\left(s_{p, q}\right)\right| .
$$

\section{Denote}

$$
\bar{w}_{n}(x)=\left(w_{10 n}(x), \ldots, w_{k 0 n}(x), w_{11 n}(x), \ldots, w_{k 1 n}(x), \ldots, w_{k(n-1) n}(x)\right)
$$

and define $\bar{w}_{n}^{*}(x)$ similarly. We observe that

$$
\varphi_{n}(x)=\bar{w}_{n}(x)\left(I_{n}-\bar{K}_{n}\right)^{-1} \bar{f}+f(x)
$$

and

$$
\varphi_{n}^{*}(x)=\bar{w}_{n}^{*}(x)\left(I_{n}-\bar{K}_{n}^{*}\right)^{-1} \bar{f}+f(x),
$$

where $I_{n}$ denotes the $(k n) \times(k n)$ identity matrix. Hence,

$$
\begin{aligned}
\varphi_{n}(x)-\varphi_{n}^{*}(x)= & \bar{w}_{n}(x)\left[\left(I_{n}-\bar{K}_{n}\right)^{-1} \bar{f}-\left(I_{n}-\bar{K}_{n}^{*}\right)^{-1} \bar{f}\right] \\
& +\left[\bar{w}_{n}(x)-\bar{w}_{n}^{*}(x)\right]\left(I_{n}-\bar{K}_{n}^{*}\right)^{-1} \bar{f} \\
= & \bar{w}_{n}(x)\left(I_{n}-\bar{K}_{n}\right)^{-1}\left(\bar{K}_{n}-\bar{K}_{n}^{*}\right)\left(I_{n}-\bar{K}_{n}^{*}\right)^{-1} \bar{f} \\
& +\left[\bar{w}_{n}(x)-\bar{w}_{n}^{*}(x)\right] \bar{\varphi}_{n}^{*} .
\end{aligned}
$$

It follows from (4.18) that

$$
\left\|\varphi_{n}-\varphi_{n}^{*}\right\|_{\infty} \leq C_{1}\left\|\bar{K}_{n}-\bar{K}_{n}^{*}\right\|_{\infty}+C_{2} \sup _{x \in[0,1]} \sum_{i=1}^{k} \sum_{j=0}^{n-1}\left|w_{i j n}(x)-w_{i j n}^{*}(x)\right| .
$$

Since $w_{i j n}$ and $w_{i j n}^{*}$ are $\alpha$-Hölder continuous on $[0,1]$, there exists an $x_{0} \in$ $[0,1]$ such that

$$
\left|w_{i j n}\left(x_{0}\right)-w_{i j n}^{*}\left(x_{0}\right)\right|=\sup _{x \in[0,1]}\left|w_{i j n}(x)-w_{i j n}^{*}(x)\right| .
$$


Combining (4.10), (4.18), (4.19), (4.20), and the above equation, we obtain

$$
\left\|\varphi_{n}-\varphi_{n}^{*}\right\|_{\infty}=C n^{-2 k+1},
$$

where $C$ is a constant independent of $n$. This completes the proof.

Corollary 4.2. Let $\varphi$ and $\varphi_{n}^{*}$ be the solutions of (4.3) and (4.12), respectively. Then $\left\|\varphi-\varphi_{n}^{*}\right\|_{\infty}=\mathscr{O}\left(n^{-k}\right)$.

Proof. Schneider [15] showed that $\left\|\varphi-\varphi_{n}\right\|_{\infty}=\mathscr{O}\left(n^{-k}\right)$. Hence, using $\left\|\varphi-\varphi_{n}^{*}\right\|_{\infty} \leq\left\|\varphi-\varphi_{n}\right\|_{\infty}+\left\|\varphi_{n}-\varphi_{n}^{*}\right\|_{\infty}$ and Theorem 4.1, we obtain the desired result.

If the points $s_{i, j}$ are selected as the zeros of the Legendre polynomials transformed into $\left[x_{j, n}, x_{j+1, n}\right]$, Theorem 3 of [15] yields the following superconvergence result.

Corollary 4.3. Let $\varphi$ and $\varphi_{n}^{*}$ be the solutions of (4.3) and (4.12), respectively. If in (4.4) we let $q=\frac{\alpha+k+2}{\alpha+\beta+1}$ for $\beta \in[\alpha+1, k+1]$, or in the logarithmic case $q=\frac{\alpha+k+2}{\alpha+\beta+1-\varepsilon}$ with $0<\varepsilon<|\alpha|$, then

$$
\left\|\varphi-\varphi_{n}^{*}\right\|= \begin{cases}\mathscr{O}\left(n^{-k+\alpha}\right) & \text { for }-1<\alpha<0, \\ \mathscr{O}\left(n^{-k-1} \log n\right) & \text { in the logarithmic case. }\end{cases}
$$

In the following example, $\alpha=-\frac{1}{2}$ and $\beta=\frac{1}{2}$, so that $\alpha+\beta+1=1$.

Example 4.1. Consider

$$
\varphi(x)-\int_{0}^{1} \frac{\varphi(y)}{\sqrt{|x-y|}} d y=f(x), \quad 0 \leq x \leq 1,
$$

where $f$ is selected so that $\varphi(x)=\sqrt{x(1-x)}$ is the solution. Piecewise linear $(k=2)$ and quadratic $(k=3)$ polynomials are used. Select $\left\{x_{j, n}\right\}_{j=0}^{n}$ according to (4.4) with $q$ described in Corollary 4.3, and let $\left\{s_{i, j}\right\}_{i=1}^{k}$ be the zeros of the Legendre polynomial of degree $k$ transformed into $\left[x_{j, n}, x_{j+1, n}\right]$. It is necessary to compute

$$
\int_{x_{j, n}}^{x_{j+1, n}} \frac{l_{i j}(y)}{\sqrt{\left|s_{i j}-y\right|}} d y \text { for } 1 \leq i \leq k, 0 \leq j \leq n-1 .
$$

These integrals are approximated by the quadrature scheme of Theorem 3.1 with $\alpha=-\frac{1}{2}$ and $S=\left\{s_{i j}\right\}$. Table 1 lists $\max _{i, j}\left|\varphi\left(s_{i j}\right)-\varphi_{n}^{*}\left(s_{i j}\right)\right|$.

\section{TABLE 1}

$n$ linear decay exp. quadratic decay exp.

$\begin{array}{ccccc}16 & 8.52 E-3 & & 1.98 E-4 & \\ 32 & 1.66 E-3 & 2.36 & 2.00 E-5 & 3.31 \\ 64 & 3.15 E-4 & 2.40 & 1.89 E-6 & 3.40 \\ 128 & 5.73 E-5 & 2.46 & 1.72 E-7 & 3.46\end{array}$




\section{ACKNOWLEDGMENT}

The authors would like to thank a referee for his comments and for bringing the paper [18] of Stenger to their attention.

\section{BIBLIOGRAPHY}

1. P. M. Anselone, Collectively compact operator approximation theory and applications to integral equations, Prentice Hall, Englewood Cliffs, NJ, 1971.

2. K. E. Atkinson, An introduction to numerical analysis, 2nd ed., Wiley, New York, 1989.

3. P. J. Davis and P. Rabinowitz, Methods of numerical integration, 2nd ed., Academic Press, Orlando, Florida, 1984.

4. I. G. Graham, Singularity expansions for the solutions of the second kind Fredholm integral equations with weakly singular convolution kernels, J. Integral Equations 4 (1982), 1-30.

5. E. Hopf, Mathematical problems of radiative equilibrium, Stechert-Hafner Service Agency, New York, 1964.

6. $\mathrm{H}$. Kaneko, $\mathrm{R}$. Noren, and $\mathrm{Y} . \mathrm{Xu}$, On the regularity of the solution of the weakly singular Hammerstein equations, Integral Equations Operator Theory 13 (1990), 660-670.

7. J. G. Kirkwood and J. Riseman, The intrinsic viscosities and diffusion constants of flexible macromolecules in solution, J. Chem. Phys. 16 (1948), 565-573.

8. R. Kress, Linear integral equations, Appl. Math. Sci., vol. 82, Springer-Verlag, Berlin, 1989.

9. D. S. Lubinsky and P. Rabinowitz, Rates of convergence of Gaussian quadrature for singular integrands, Math. Comp. 47 (1984), 219-242.

10. R. Piessens and M. Branders, Numerical solution of integral equations of mathematical physics using Chebyshev polynomials, J. Comp. Phys. 21 (1976), 178-196.

11. P. Rabinowitz and I. H. Sloan, Product integration in the presence of a singularity, SIAM J. Numer. Anal. 21 (1984), 149-166.

12. J. R. Rice, On the degree of convergence of nonlinear spline approximation, Approx. with Emphasis on Spline Functions (I. J. Schoenberg, ed.), Academic Press, New York, 1969, pp. 349-365.

13. G. Richter, On weakly singular Fredholm integral equations with displacement kernels, J. Math. Anal. Appl 55 (1976), 32-42.

14. C. Schneider, Regularity of the solution to a class of weakly singular Fredholm integral equations of the second kind, Integral Equations Operator Theory 2 (1979), 62-68.

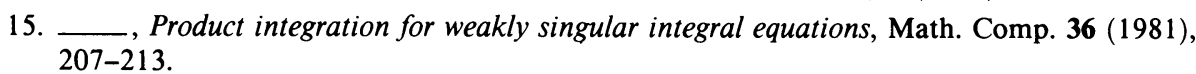

16. I. H. Sloan, On the numerical evaluation of singular integrals, BIT 18 (1978), 91-102.

17. I. H. Sloan and W. E. Smith, Product-integration with the Clenshaw-Curtis and related points, Numer. Math. 30 (1978), 415-428.

18. F. Stenger, Numerical methods based on Whittaker cardinal, or sinc quadrature, SIAM Rev. 23 (1981), 165-224.

19. M. H. Taibleson, On the theory of Lipschitz spaces of distributions on Euclidean n-space. I, J. Math. Mech. 13 (1964), 407-479; II, J. Math. Mech. 14 (1965), 813-839.

Department of Mathematics and Statistics, Old Dominion University, Norfolk, VirGINIA 23529-0077

E-mail address: kaneko@math.odu.edu 58105

Department of Mathematics, North Dakota State University, Fargo, North Dakota

E-mail address: xu@plains. NoDak.edu 